\title{
Significant broadband extinction abilities of bioaerosols
}

\author{
Yihua $\mathrm{Hu}^{1,2}$, Xinying Zhao ${ }^{1,2^{*}}$, Youlin $\mathrm{Gu}^{1,2}$, Xi Chen ${ }^{1,2}$, Xinyu Wang ${ }^{1,2}$, Peng Wang ${ }^{3}$, \\ Zhiming Zheng ${ }^{3}$ and Xiao Dong ${ }^{1,2}$
}

\begin{abstract}
Bioaerosol, an important constituent of the atmosphere, can directly affect light radiation characteristics due to absorption and scattering effects. Current research lacks a reasonable explanation for the extinction abilities of bioaerosols in a broadband. Herein, we measured the reflectance spectra of 12 common biomaterials and calculated their complex refractive indexes. The peaks of the imaginary part of the complex refractive indexes are located at wavelengths of approximately $0.7,2.7,6.1$ and $9.5 \mu \mathrm{m}$. Based on photographs of the floating structures of bioaerosols, we constructed a model for calculating the extinction abilities of bioaerosols in the wavelength range of $240 \mathrm{~nm}$ to $14 \mu \mathrm{m}$. Taking AN02 spores as an example, absorption was found to account for more than $\mathbf{9 0 \%}$ of the total extinction. In addition, the theoretical calculations and experimental data of transmittance corresponding to the smoke box show that bioaerosol exhibits significant broadband extinction ability from UV to IR bands, which provides new directions for the development of broadband light attenuation materials.
\end{abstract}

Keywords: bioaerosol, complex refractive index, UV to IR, broadband, light attenuation

\section{INTRODUCTION}

Bioaerosols, a subset of atmospheric particles, are directly emitted from terrestrial and marine ecosystems or are artificially released into the atmosphere [1-4]. They comprise living and dead organisms, dispersal units, and various fragments or excretions [5-7]. Typical bioaerosol particles are bacteria, viruses, spores, fungi, algae, protozoa and pollens, ranging in size from $<10 \mathrm{~nm}$ to $\sim 100 \mu \mathrm{m}$ [8-10]. Bioaerosols are widely distributed on global or local scale. According to the data given by MatthiasMaser et al. [11] in 2000, bioaerosols accounted for 28\%,
$22 \%$ and $10 \%$ of the suspended particles in the air in distant continents, densely populated continents and distant marine environments, respectively. In the tropics, $16 \%-80 \%$ of the major aerosols come from biological sources [12]. A study by Huffman et al. [13] suggested that over the Amazon rainforest, the number and mass concentrations of bioaerosols with diameters greater than $1 \mu \mathrm{m}$ were $7.3 \times 10^{4} \mathrm{~m}^{-3}$ and $0.72 \mu \mathrm{g} \mathrm{m}^{-3}$, respectively, accounting for $24 \%$ of the total particle number and $47 \%$ of the total particle mass.

Bioaerosols in the atmosphere affect light transmission, which changes atmospheric radiation characteristics [14,15]. Spänkuch et al. [16] found that when the concentration of pine pollen increased, the infrared (IR) flux decreased significantly, indicating that some pollen emissions may lead to local atmospheric warming events. Another study showed that up to $47 \%$ of the light absorption over the Amazon rainforest was attributed to bioaerosols in the atmosphere during the wet season and up to $35 \%$ during the wet-to-dry transition period [17]. On the other hand, bioaerosols have an impact on the utilization of light waves. Optical sensors cannot evade bioaerosols when they are operating in the atmosphere. The extinction characteristics of bioaerosols directly affect the accuracy of optical remote sensing, light detection, optical communication and other activities [18-23]. Bioaerosols can affect the effective working range of lidars. Makogon [18] found that the range of operation of the lidar at an aerosol concentration of $100 \mathrm{~cm}^{-3}$ was more than $30 \mathrm{~km}$ (aerosol IR channel) and $4 \mathrm{~km}$ (fluorescent UV channel). Christesen et al. [19] tested the UVLIDAR Fluorosensor system against the aerosolized bacterial spore Bacillus subtilus var. niger sp. globiggi (BG) and several likely interferences at several ranges from

\footnotetext{
${ }^{1}$ State Key Laboratory of Pulsed Power Laser Technology, National University of Defense Technology, Hefei 230037, China

${ }^{2}$ Anhui Province Key Laboratory of Electronic Restriction, National University of Defense Technology, Hefei 230037, China

${ }^{3}$ Key Laboratory of Ion Beam Bioengineering, Hefei Institutes of Physical Science, Chinese Academy of Sciences, Hefei 230001, China

* Corresponding author (email: dd_321@sina.com)
} 
approximately 600 to $3,000 \mathrm{~m}$. Their tests with BG indicate a detection limit of approximately $500 \mathrm{mg} \mathrm{m}^{-3}$ at a range of $3,000 \mathrm{~m}$. The extinction characteristics of bioaerosols affect the operating band of optical sensors. Yabushita et al. $[20,21]$ studied the extinction of microorganisms in interstellar dust in the ultraviolet, visible, and IR band, showing that the well-known $220 \mathrm{~nm}$ peak in the extinction of starlight was explained by a mixed culture of diatoms and bacteria [22]. This result reminds us of choosing a working band that is not absorbed by bioaerosols when using optical means to detect interstellar objects. The content of bioaerosol in the atmosphere will affect the accuracy of atmospheric remote sensing. Ligon et al. [23] found that the spectral characteristics of composite clouds containing bioaerosols and pure clouds without bioaerosols were different when passive remote sensing detected the optical properties of atmospheric aerosols. However, this difference is only apparent when the bioaerosol content is large. Therefore, studying the extinction characteristics of bioaerosols is beneficial to our comprehensive understanding of atmospheric radiation characteristics, and recognizing the adverse effects of bioaerosols on optical sensors which should be considered to improve optical sensor design.

The bioaerosols in the atmosphere are mixed with other substances, and it is challenging to analyze their extinction abilities alone. Therefore, some researchers have used laboratory-cultivated pure biomaterials to generate bioaerosols and performed quantitative experiments on their extinction characteristics. Gittins et al. [24] measured the extinction coefficients for aerosolized BG spores at $2.7-12 \mu \mathrm{m}$, and the measurements indicated a peak extinction coefficient of approximately $1.6 \times 10^{-8} \mathrm{~cm}^{2}$ per spore at $9.65 \mu \mathrm{m}$. Gurton et al. [25] measured the spectral extinction in situ for aerosolized BG spores using Fourier transform infrared spectroscopy for sizes from 3 to $13 \mu \mathrm{m}$ and then explained the experimental transmission data based on the Mie theory calculation and the available refractive index data. Yabushita and Wada [26] also mentioned that it was challenging to reasonably explain all the extinction features from the UV to IR bands in terms of a single substance, whether of inorganic, organic or microorganism origin.

Although the study on the extinction abilities of bioaerosols has advanced from qualitative to quantitative research, the following problems still remain to be solved. Most previous studies have focused only on the extinction abilities of bioaerosols in a single band, particularly in the IR bands. In stark contrast, bioaerosols in the atmosphere absorb and scatter the entire spectrum of solar radiation, and little attention has been paid to this mechanism, which may lead to an incomplete understanding of the extinction characteristics of bioaerosols. On the other hand, the current quantitative analysis of the extinction abilities of bioaerosols depends mostly on the experimental results and lacks reasonable theoretical explanation, which is a consequence of the challenges involved in the calculation of the electromagnetic attenuation characteristics of bioaerosols due to their various forms. When bioaerosols float in the atmosphere, the particles collide, rub against and adsorb to each other, resulting in static electricity and agglomeration. This agglomeration phenomenon enriches the particle structure of bioaerosols, which is no longer limited to the shape of the original particles [6]. Studies have shown that when BG spores float in the air, the structure may consist of single or unattached organisms, or clusters of many particles, each containing 2 to 10 primary particles [26]. The shape of the agglomerated particles is far from spherical, and Mie scattering theory is not suitable to describe the scattering effect of bioaerosols. Thus, it is necessary to apply a new theory to describe the extinction mechanism of bioaerosols.

Here, we calculated the complex refractive indexes of bioaerosols and summarized the scattering and absorption characteristics of bioaerosols in the UV to IR regions of the electromagnetic spectrum. The spatial structure of bioaerosols in the floating state was imaged by a threedimensional microscopy system with a superwide depth of field. We used the discrete-dipole approximation (DDA) model to calculate the extinction abilities of bioaerosols and analyzed the phenomena that most strongly influence the extinction effect.

\section{MATERIALS AND METHODS}

Twelve biomaterials were provided by the Key Laboratory of Ion Beam Bioengineering, Chinese Academy of Sciences. They were isolated by the laboratory and stored at $-80^{\circ} \mathrm{C}$ in sterilized cryovials containing $10 \%$ glycerol (in $0.02 \%$ Tween- 80 solution). We optimized the fermentation conditions to improve the collection efficiency for the biomaterials production [27-29]: bacterial species activation $\rightarrow$ shake flask culture $\rightarrow$ large-scale tank fermentation $\rightarrow$ centrifugation $\rightarrow$ pure water cleaning $\rightarrow$ vacuum freeze-drying $\rightarrow$ crushing using a ultra-fine Chinese medicine crusher (See the supplementary information, text section for specific steps). And then the biomaterials were stored in desiccators containing silica gel absorbent (Fig. S1), sealed and bagged at room temperature (Fig. S2). 
Twelve tablets of common bioaerosol materials were fabricated for spectral reflectance measurements (Fig. S3). In the experiment, the pressure of the tablet machine was kept at $20 \mathrm{MPa}$, and the holding time was $3 \mathrm{~min}$ (Fig. S4a). The weight of each tablet was fixed at $2.5 \mathrm{~g}$. The tablets had a diameter of $4 \mathrm{~cm}$ and thickness of $0.5-1.5 \mathrm{~mm}$, and the surface was as smooth as possible (Fig. S4b). In the $240 \mathrm{~nm}$ to $2.5 \mu \mathrm{m}$ band, the reflectance was measured using a spectrophotometer (Hitachi U4100, Hitachi, Ltd., Tokyo, Japan, Fig. S5a). Base calibration was performed using a $1 \mathrm{~cm}$ thick $\mathrm{BaSO}_{4}$ cylinder. In the 2.5 to $14 \mu \mathrm{m}$ band, the reflectance was measured using a Fourier transform infrared spectrometer (MAGNA-IR 750, Nicolet Instrument Co. USA) with a microscope (Continu $\mu \mathrm{m}$, Nicolet Instrument Co. USA) and a gold-plated mirror used as a substrate (Fig. S5b). Fig. 1a and $b$ show the operational principles of the reflection spectrum measurements in the $240 \mathrm{~nm}$ to $2.5 \mu \mathrm{m}$ and 2.5 to $15 \mu \mathrm{m}$ ranges, respectively. Fig. $1 \mathrm{c}$ and $\mathrm{d}$ show the reflectivity data of the corresponding bands. The reflectivity measured by the spectrophotometer is not the same as that measured by Fourier infrared microscopy at $2.5 \mu \mathrm{m}$, which is due to the use of different measurement methods. The reflectance at $2.5 \mu \mathrm{m}$ was obtained by averaging the reflectance obtained by the spectrophotometer and Fourier infrared microscopy.

\section{Complex refractive index calculation model}

Based on the measured reflection spectra, the complex refractive index values of the bioaerosols in the $240 \mathrm{~nm}-14 \mu \mathrm{m}$ wavelength range were calculated using the Kramers-Kronig (K-K) relation [30-33]. Our group have previously used this method for calculating the complex refractive index of biomaterials [27,34-44]. According to this relation, the reflective phase shift $\Theta(\lambda)$ can be expressed as [45]

$$
\Theta(\lambda)=\frac{\lambda}{\pi} P \int_{0}^{\infty} \frac{\ln R\left(\lambda^{\prime}\right)}{\left(\lambda^{\prime 2}-\lambda^{2}\right)} \mathrm{d} \lambda^{\prime},
$$

where $R(\lambda)$ is the vertical reflectivity, $P$ is the Cauchy principal value function, and $\lambda$ is the wavelength.

The real parts $(n)$ and imaginary parts $(k)$ of the complex refractive can be obtained as [29]

$$
\begin{aligned}
& n(\lambda)=\frac{R(\lambda)}{1+R(\lambda)+2 \sqrt{R(\lambda)} \cos \Theta(\lambda)}, \\
& k(\lambda)=\frac{2 \sqrt{R(\lambda)} \cos \Theta(\lambda)}{1+R(\lambda)+2 \sqrt{R(\lambda)} \cos \Theta(\lambda)} .
\end{aligned}
$$

It is known that the $R(\lambda)$ should be obtained in the full band to calculate the complex refractive index. However, in actual experimental measurements, the $R(\lambda)$ can be measured only in the limited band ( $240 \mathrm{~nm}$ to $14 \mu \mathrm{m})$. The constant extrapolation method [45] was used to expand the range of reflectivity. A value of $100 \mu \mathrm{m}$ was used as the upper limit of the integral because the reflectivity above $100 \mu \mathrm{m}$ had little effect on the complex refractive index in the $240 \mathrm{~nm}$ to $14 \mu \mathrm{m}$ wavelength range. $R(0-$ $240 \mathrm{~nm})=R(240 \mathrm{~nm})$, and $R(14-100 \mu \mathrm{m})=R(14 \mu \mathrm{m})$; therefore, $R(\lambda)$ over the entire integral range $(0-100 \mu \mathrm{m})$ was acquired.

\section{Computing the aggregated particles of bioaerosols}

Based on the micrographs of the floating bioaerosols, we assumed that the original bioaerosol particle of aggregated particles was spherical. The aggregated geometry was computed using the CCA code described in previous work [46].

\section{Extinction calculation model for bioaerosol aggregated particle swarm}

The DDA method uses an array of discrete interacting small dipoles to approximate the actual particles of bioaerosols. These small dipoles must describe the shape and electromagnetic properties of the simulated particles as accurately as possible, which means that they have the same dispersion relationship, so the results obtained for the actual particles can be converted into the results obtained by the study of these small dipoles $[47,48]$. DDA can be applied to particles of any geometric shape as long as the condition $|m-1| \lesssim 3$ is satisfied [45]. According to Fig. 1 and references [49-51], particularly formula (6), the equivalent complex refractive index $m_{\text {eff }, \lambda}$ of the bioaerosol particles meets the required conditions of DDA. We used DDA to calculate the broadband extinction characteristics of bioaerosols with complex structures.

We used the following parameters to describe the geometry of an aggregated bioaerosol particle. The radius of the original bioaerosol particle is denoted by $r$, the number of original particles contained in each aggregated bioaerosol particle is denoted by $N$, the porosity of the aggregated particle is denoted by $P$, the radius of gyration is denoted by $R_{\mathrm{g}}$, and the complex refractive index of the biomaterial at incident light $\lambda$ is denoted by $m_{\lambda}$. The relationship between $N$ and $R_{\mathrm{g}}$ is approximated as [52]

$R_{\mathrm{g}}^{2}=\frac{1}{2 N^{2}} \sum_{i=1}^{N} \sum_{j=1}^{N}\left|r_{i}-r_{j}\right|^{2}$,

where $r_{i}$ and $r_{j}$ are the coordinates in space of the $i$ th and $j$ th original particles, respectively. The porosity of the 

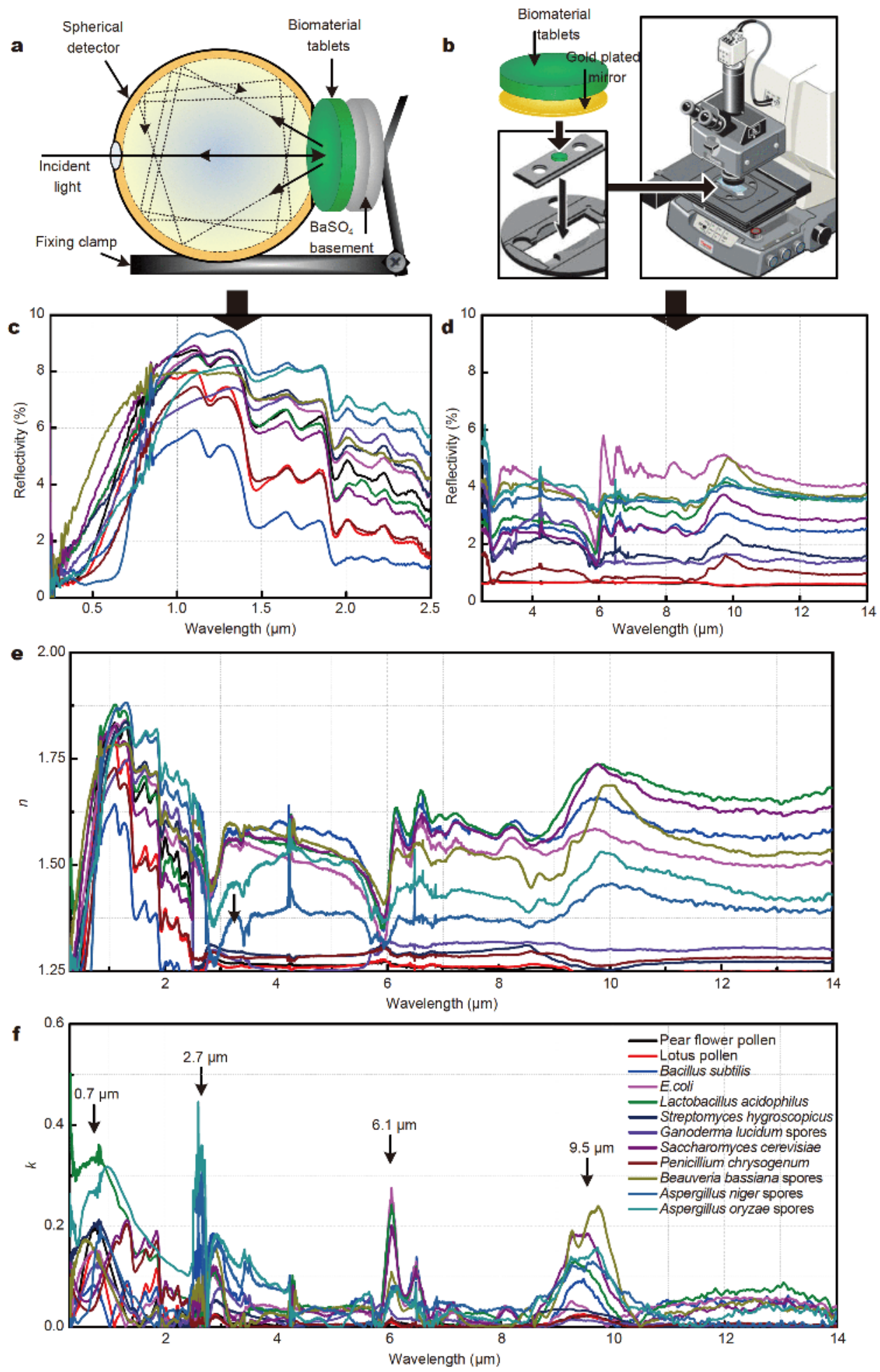

Figure 1 Complex refractive indexes of the bioaerosols. (a) Operational principle of the measurement of the reflection spectrum in a spectrophotometer. The $\mathrm{BaSO}_{4}$ base is removed in the real tests and is shown here for illustration only. (b) Schematic diagram of installation of biotablets and a gold-plated mirror on the microscope stage. (c) Reflectivity in the $240 \mathrm{~nm}$ to $2.5 \mu \mathrm{m}$ wavelength range. (d) Reflectivity in the 2.5 to $14 \mu \mathrm{m}$ wavelength range. (e) Real parts of the complex refractive indexes of bioaerosols. (f) Imaginary parts of the complex refractive indexes of bioaerosols. Absorption peak locations are marked by arrows in the figure. ( $c-f)$ share the same legend as marked in the upper right corner of (f). 
aggregated particles is given by $[53,54]$

$P=1-N\left[r /\left(\sqrt{5 / 3} R_{\mathrm{g}}\right)\right]^{3}$.

The equivalent refractive index $m_{\mathrm{eff}, \lambda}$ of the aggregated particle with the porosity $P$ is [55]

$m_{\mathrm{eff}, \lambda}^{2}=1+\frac{3(1-P)\left(m_{\lambda}{ }^{2}-1\right) /\left(m_{\lambda}{ }^{2}+2\right)}{1-(1-P)\left(m_{\lambda}{ }^{2}-1\right) /\left(m_{\lambda}{ }^{2}+2\right)}$,

where $P$ reflects the structural characteristics of the bioaerosol aggregated particles, while $m_{\lambda}$ reflects the compositional characteristics. In the following calculations, replacing $m_{\lambda}$ with $m_{\text {eff } \lambda}$ makes the calculation results more accurate [56].

Equation (6) was used in the DDA algorithm to calculate the absorption cross section and the scattering cross section of a single bioaerosol aggregated particle as given by [57]

$$
\begin{aligned}
& C_{\mathrm{abs}}=\frac{4 \pi k}{\left|E_{0}\right|^{2}} \sum_{j=1}^{N}\left\{\operatorname{Im}\left[\overrightarrow{P_{j}} \cdot\left(\alpha_{j}^{-1}\right)^{*} \cdot \overrightarrow{P_{j}^{*}}\right]-\frac{2}{3} k^{3}\left|\overrightarrow{P_{j}}\right|^{2}\right\}, \\
& C_{\mathrm{sca}}=\frac{k^{4}}{\left|\overrightarrow{E_{\mathrm{inc}}}\right|^{2}} \times \int \mathrm{d} \Omega\left[\sum_{j=1}^{N}\left[\overrightarrow{P_{j}}-\vec{n}\left(\vec{n} \cdot \overrightarrow{P_{j}}\right)\right] \exp \left(-i k \vec{n} \cdot \overrightarrow{r_{j}}\right)\right]^{2},
\end{aligned}
$$

where $k=2 \pi / \lambda, \lambda$ is the wavelength of incident light; $E_{0}$ and $E_{\text {inc }}$ are the electric field strength vectors; $\overrightarrow{P_{j}}=\left(4 \pi r^{3} / 3\right) p_{j}, \overrightarrow{p_{j}}$ is the polarization strength vector; $\alpha_{j}$ is the polarity, $\alpha_{j}=4 \pi r^{3} \varepsilon_{0}\left[\left(n_{\lambda}^{2}-1\right) /\left(n_{\lambda}^{2}+2\right)\right]$, $\varepsilon_{0}$ is the complex dielectric coefficient; $\mathrm{d} \Omega$ indicates the solid angle microelements; $\vec{n}$ is the unit vector of the scattering direction; $r_{j}^{\prime}$ is the center of the integration area; superscript * represents the complex conjugate; and Im is the complex imaginary part.

We assumed that the density of the bioaerosol aggregated particle swarm was $\rho$ and that all aggregated particles were randomly oriented and evenly distributed in the particle swarm. The absorption cross section $C_{\text {abs }}$ and scattering cross section $C_{\mathrm{sca}}$ of a single bioaerosol aggregated particle can be calculated by Equations (7 and 8 ). The optical path through the swarm is $L$. Taking all the above-described parameters, the Monte Carlo algorithm $[58,59]$ can be used to calculate the transmittance $T$ of incident light through the bioaerosol aggregated particle swarm.

Transmittance experiment of bioaerosols in the smoke box We built a smoke box for the transmittance experiment $(4 \mathrm{~m} \times 3 \mathrm{~m} \times 2.4 \mathrm{~m})$. Light sources and detectors for different wave bands were placed on both sides of the smoke box. The light sources and detector modes with the spectral range are shown in Table 1 . The light path between the light source and the corresponding detector was $4 \mathrm{~m}$. Forty milligram of each kind of biological materials was weighed with an electronic balance and filled in the filling port of the smoke box. $\mathrm{N}_{2}$ gas (10 MPa) was used to inject the biomaterial into the smoke box. The valve switch of $\mathrm{N}_{2}$ was closed after all the biomaterial was sprayed in the smoke box. Two fans located on the diagonal inside the smoke box were used to accelerate the dispersion of the biological material. When the biomaterial in the smoke box was evenly distributed, the average power of the light signal received by the detector was recorded. The ratio of the average received light power to the initial emission light power was regarded as the average transmittance of the target wave band. On the basis of plentiful experimental results, it can be estimated that approximately $60 \%-80 \%$ of the initial mass of the biomaterials stably dispersed in the smoke box. Taking the material loss into account, theoretically equivalent mass and structure of these three biomaterials that were actually dispersed in the smoke box were included in the extinction calculation model.

\begin{tabular}{|c|c|c|c|c|}
\hline \multirow{2}{*}{$\begin{array}{c}\text { Experimental } \\
\text { measurement band }\end{array}$} & \multicolumn{2}{|c|}{ Light source } & \multicolumn{2}{|c|}{ Detector } \\
\hline & Light source model & Emission spectral range & Detector model & $\begin{array}{l}\text { Response spectra } \\
\text { range }\end{array}$ \\
\hline UV band & $\begin{array}{l}\text { NBeT Merc-500 } \\
\text { mercury lamp }\end{array}$ & $180-500 \mathrm{~nm}$ & $\begin{array}{c}\text { Newport } 843-\mathrm{R} \\
\text { (Probe model } 818 \mathrm{UV} \text { ) }\end{array}$ & $200-1,100 \mathrm{~nm}$ \\
\hline Visible light band & NBeT PS230 & $350-700 \mathrm{~nm}$ & $\begin{array}{c}\text { OPHIR starlite } \\
\text { (Probe model PD300) }\end{array}$ & $350-1,100 \mathrm{~nm}$ \\
\hline Mid-infrared band & $\begin{array}{c}\text { Fuyuan black body } \\
\text { HFX-300A }\end{array}$ & $\begin{array}{c}\text { Temperature } \\
5-400^{\circ} \mathrm{C}\end{array}$ & FLIR SC7000 & $1.5-5.1 \mu \mathrm{m}$ \\
\hline Far-infrared band & $\begin{array}{c}\text { Fuyuan black body } \\
\text { HFX-300A }\end{array}$ & $\begin{array}{c}\text { Temperature } \\
5-400^{\circ} \mathrm{C}\end{array}$ & FLIR SC7900VL & $7.8-12 \mu \mathrm{m}$ \\
\hline
\end{tabular}

Table 1 Light sources and detector modes used in the experiment 


\section{RESULTS}

\section{Complex refractive indexes of bioaerosols}

The complex refractive index $m$ is an important optical constant for describing the optical properties of bioaerosols, and its imaginary part $k$ corresponds to the absorption coefficient. We calculated the complex refractive indexes of bioaerosols using the K-K relationship [30-32] with the reflection spectra of 12 kinds of bioaerosols in the $240 \mathrm{~nm}$ to $14 \mu \mathrm{m}$ band (Fig. 1e, f). Since the UV to NIR spectrophotometer and the Fourier infrared spectrometer have slight jitter instability at the beginning of each test, the initial portion of the reflectance curves in Fig. $1 \mathrm{c}$ and $\mathrm{d}$ fluctuate. Therefore, the calculation error of the complex refractive index in the corresponding small band increases. The real index $n$ of bioaerosol particles ranges from 1.250 to 1.875 (Fig. 1e), and the imaginary index $k$ ranges from 0 to 0.4 (Fig. 1f). As shown in Fig. 1f, the absorption spectra of 12 types of biomaterials have similar shapes, with the absorption peaks overlapping each other and concentrating at $0.7 \mu \mathrm{m}\left(14,285 \mathrm{~cm}^{-1}\right)$, $2.7 \mu \mathrm{m}\left(3,703 \mathrm{~cm}^{-1}\right), \quad 6.1 \mu \mathrm{m} \quad\left(1,639 \mathrm{~cm}^{-1}\right) \quad$ and $9.5 \mu \mathrm{m}\left(1,050 \mathrm{~cm}^{-1}\right)$, which is in agreement with the results shown in Fig. S6. The origins of the appearance of the common absorption peaks of bioaerosols are analyzed in the discussion section.

\section{Bioaerosol spatial structure}

Fig. 2 shows the micrographs of AN02 spores, BB01 spores and $\mathrm{AO} 03$ spores when these spores are floating in the air (1,000× magnification). The micrographs shown in Fig. 2b-d (Fig. S7b-d) were obtained by a three-dimensional microscopy system with a superwide depth of field (Fig. 2a, Fig. S7a, KEYENCE VHX-6000, the camera has a standard resolution of $1600(\mathrm{H})+1200(\mathrm{~V})$ and a maximum frame rate of $50 \mathrm{~F} \mathrm{~s}^{-1}$ ). The bioaerosol particles agglomerate due to the collisions and friction and form aggregated particle swarms, further contributing to the diversity of bioaerosol particle structures. The number of primary particles included in agglomerated particles varies from a few to a few hundreds. The structure of bioaerosol aggregated particles in the actual floating state was simulated by the cluster-cluster aggregation (CCA) model [46] (see Methods part) as shown in Fig. 2e-g.

\section{Extinction calculation model}

The micrographs presented in Fig. $2 b-d$ show that the bioaerosols have an aggregated geometry when floating in the air. This kind of aggregated geometry enriches the size distribution of bioaerosols. To consider the aggregation of bioaerosols, we used the DDA method to calculate the extinction abilities of bioaerosols $[42,50,60,61]$ (see Methods part). Then, the transmittance of the incident light through the bioaerosol aggregated particle swarm was obtained by Monte Carlo simulation [58,59]. Assuming that AN02 spores diffuse evenly within a certain volume of space, the density $(\rho)$ of aggregated particles is approximately $200-1,000 \mathrm{~cm}^{-3}$. Each aggregated particle contains 40 original particles (Fig. S8); thus, $N=40$. The radius of the original particle is $1.5 \mu \mathrm{m}$; thus, $r=1.5 \mu \mathrm{m}$. The light path is $4 \mathrm{~m}$; thus, $L=4 \mathrm{~m}$. The extinction ability of AN02 spores in the $240 \mathrm{~nm}$ to $14 \mu \mathrm{m}$ band is shown in Fig. 3.

\section{Experimental analysis}

In the previous work, we calculated the spectral transmittance of a typical bioaerosol, AN02 spores, which displayed the broadband extinction ability in the UV to IR bands. Here, we designed a smoke box experiment for 12 kinds of bioaerosols that mentioned in Fig. 1 to test their light attenuation abilities. BB01 spores, AN02 spores and $\mathrm{AO} 03$ spores distinguished themselves from others. Electron micrographs $(20,000 \times$ magnification) of these three materials shot by scanning electron microscopy (Sirion 200, FEI, Ltd., headquartered in Hillsboro, Oregon, USA) are shown in Fig. $4 \mathrm{a}-\mathrm{c}$. BB01 spores are in the shape of pancakes with depressions in the middle and relatively smooth surface, resembling red blood cells (diameter: $2 \mu \mathrm{m}$; thickness: $0.7 \mu \mathrm{m}$; depth of the depression area: $0.5 \mu \mathrm{m}$ ). AN02 spores are squash-shaped with depressions in the middle and elongated bulges on the surface (diameter: $3-4 \mu \mathrm{m}$, thickness: $2-3 \mu \mathrm{m}$, depth of the depression area: $0.3 \mu \mathrm{m}$ ). AO03 spores are nearly spherical with the diameter of approximately 3-4 $\mu \mathrm{m}$ and hundreds of nanometer-scale protrusions on the surface. See the Method section for the experimental procedure of the smoke box. We assumed that each bioaerosol aggregated particle contains 40 primary particles (see Fig. S8) based on the pictures in Fig. 2b-d. Combined with the density value of the biological material, the density range of aggregated particles can be deduced. Here, we take $\rho=1,000 \mathrm{~cm}^{-3}$. The theoretical calculation results and actual measured transmittance values in the band of $240 \mathrm{~nm}$ to $14 \mu \mathrm{m}$ are shown in Fig. $4 \mathrm{e}-\mathrm{g}$.

\section{DISCUSSION AND CONCLUSION}

The absorption peaks of the spectra cannot be separated from the peaks of the spectra of the functional groups of bioaerosol components in the corresponding wavelength region. Water, proteins and nucleic acids are considered 

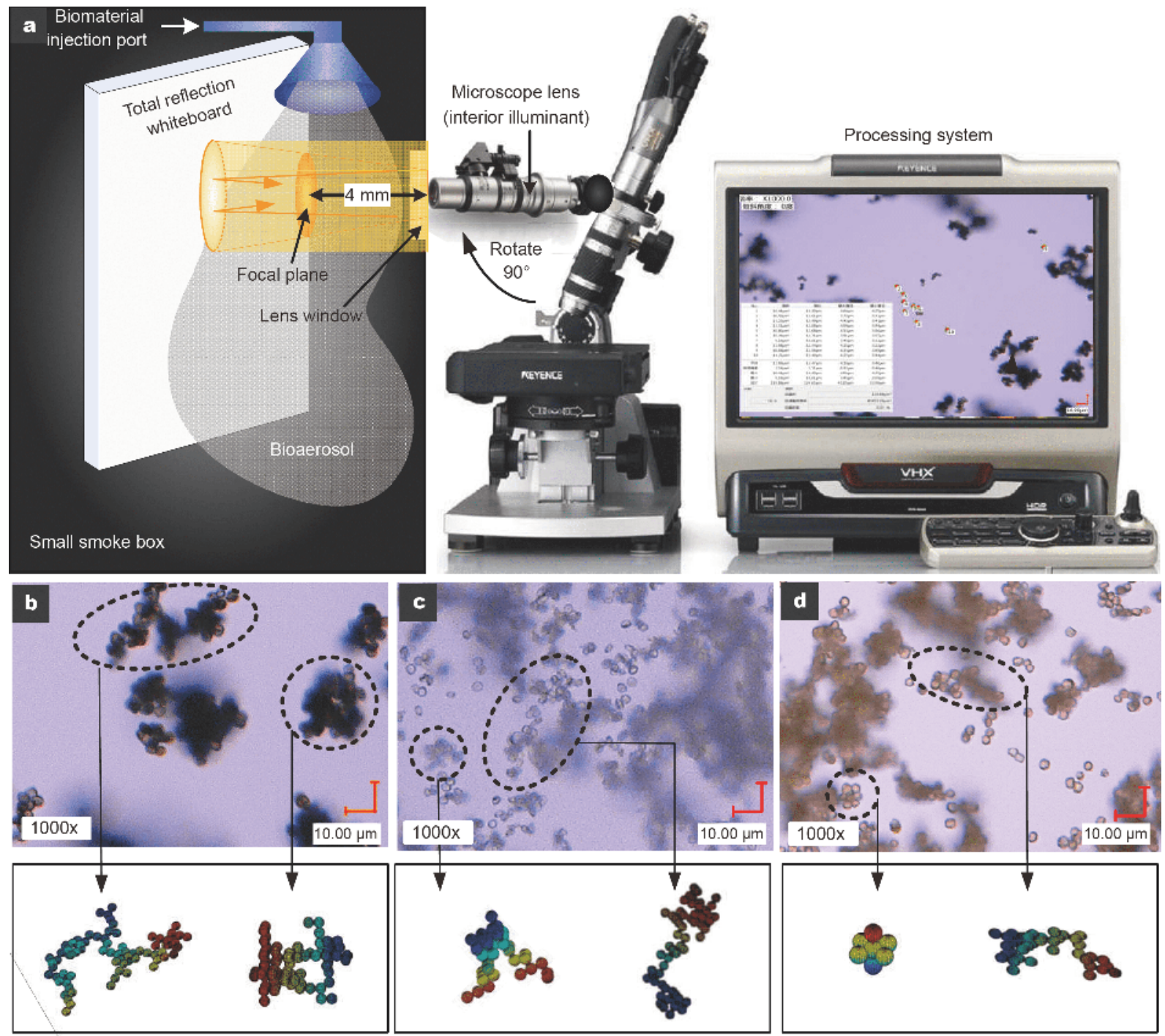

Figure 2 Micrographs of bioaerosols in the floating state. (a) An experimental system is built to obtain the micrographs of bioaerosols in the floating state. The bioaerosol is ejected from the injection port, fully dispersing in the tiny space between the total reflection whiteboard and the microscope head. When the biological particles float to the focal plane of the lens, a clear image is obtained. The focal length of the lens is $4 \mathrm{~mm}$. (b-d) Micrographs of AN02 spores, BB01 spores and AO03 spores when the spores are floating in space (1,000× magnification). When the bioaerosol particles move to the focal plane, the immediately following images are clear, and bioaerosol particles out of the focal plane are more obscure. (e-g) Aggregated particle structures constructed by the CCA model.

to be the main components of bioaerosols [62-67]. We analyzed the origins for the formation of the absorption peaks and presented the results of the analysis (Fig. S6) for the constituent functional groups. Water molecules have absorption peaks at 3,650-3,590, 3,500, 3,320 and $3,570-3,450 \mathrm{~cm}^{-1}$, which are related to the stretching vibrations of the $\mathrm{O}-\mathrm{H}$ bonds; the absorption peaks at $\sim 1,400 \mathrm{~cm}^{-1}$ are related to the bending vibrations of the $\mathrm{O}-\mathrm{H}$ bonds [62]. For proteins, in the ultraviolet region, the group consisting of single bonds has only $\sigma$ bond electrons and can produce only $\sigma-\sigma^{*}$ transitions. The transition energy of the $\sigma$ bond electrons is high, and the excitation is very challenging. The absorption spectra of single bond groups in the ultraviolet region are located in the far ultraviolet region $(\lambda<150 \mathrm{~nm})$ [63]. Therefore, the groups composed of single bonds in protein molecules, such as $-\mathrm{CH}_{2}-, \mathrm{C}-\mathrm{N},-\mathrm{CH}_{3}$, and hydrogen bonds, for example, absorb only in the far ultraviolet region. If the single-bonded group also contains $\mathrm{O}, \mathrm{N}$ and $\mathrm{S}$ atoms in addition to the $\sigma$ bond electrons in the group, this group also contains nonshared p-electron pairs that can generate $n-\sigma^{*}$ transitions, resulting in a series of absorption 

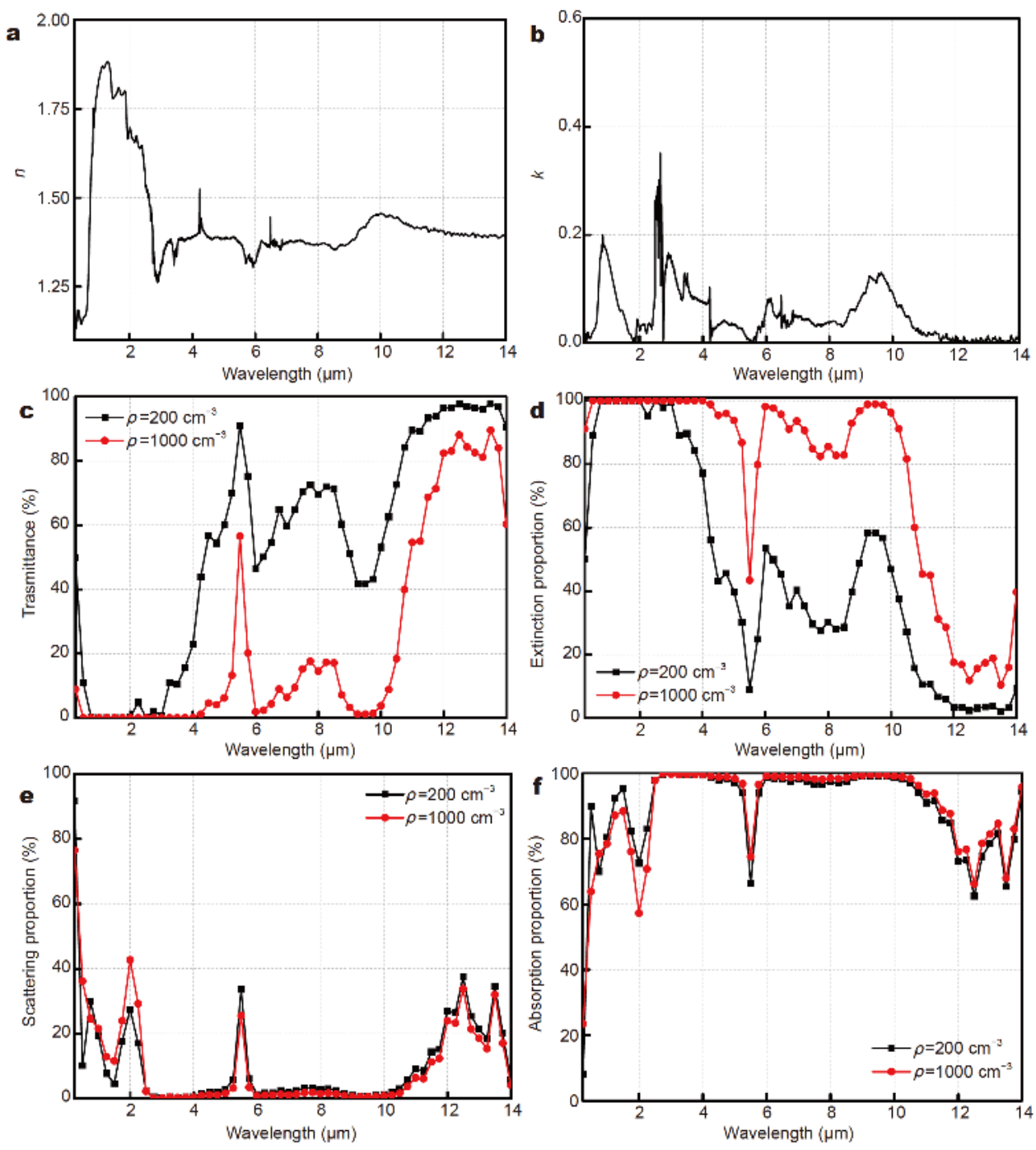

Figure 3 Calculation of the extinction abilities of AN02 spores. (a, b) Real and imaginary parts of the complex refractive index of AN02 spores in the $240 \mathrm{~nm}$ to $14 \mu \mathrm{m}$ band. (c) Transmittance of AN02 spores with incident light passing through a light path of $4 \mathrm{~m}$. The area between the black line ( $\rho=$ $\left.200 \mathrm{~cm}^{-3}\right)$ and the red line $\left(\rho=1,000 \mathrm{~cm}^{-3}\right)$ is the range of transmittance variation with the density $\left(200 \mathrm{~cm}^{-3}<\rho<1,000 \mathrm{~cm}^{-3}\right)$. (d) Extinction proportion of incident light in the $240 \mathrm{~nm}$ to $14 \mu \mathrm{m}$ band for AN02 spores. (e-f) Proportions of absorption and scattering in the extinction effect.

peaks that are close to or within the near ultraviolet region $(200-380 \mathrm{~nm})$. Therefore, the groups of $-\mathrm{NH}_{2},-\mathrm{OH}$, $\mathrm{C}-\mathrm{N}$, and $\mathrm{C}-\mathrm{S}$ in the protein molecule have absorption peaks near $200 \mathrm{~nm}$ [64]. In protein molecules, the unsaturated chemical bonds $>\mathrm{C}=\mathrm{C}<,>\mathrm{C}=\mathrm{O}$, and $>\mathrm{C}=\mathrm{N}-$ contain $\pi$-bonded electrons in addition to $\sigma$-bonded electrons, which can generate $\pi-\pi^{*}$ transitions with absorption peaks located in the ultraviolet region in the vicinity of $200 \mathrm{~nm}$. When n-electrons coexist with the $\pi$ bond in the protein molecule, $\mathrm{n}-\pi^{*}$ transitions occur, and ultraviolet light in the $200-380 \mathrm{~nm}$ band is absorbed [64]. In the IR region, the absorption peaks of protein molecules are relatively abundant. For example, the absorption peaks of the $\mathrm{O}-\mathrm{H}$ and $\mathrm{N}-\mathrm{H}$ groups are in the range of $4,000-3,000 \mathrm{~cm}^{-1}$, the absorption peaks of the $\mathrm{C}-\mathrm{H}$ group are in the range of $3,300-2,700 \mathrm{~cm}^{-1}$, and the absorption peaks of the $>\mathrm{C}=\mathrm{O}$ group are in the range of $1,900-1,650 \mathrm{~cm}^{-1}$, for example $[65,66]$. The main functional groups of nucleic acid molecules include $\mathrm{C}-\mathrm{O}-\mathrm{C}$, $-\mathrm{PO}_{4}{ }^{2-},-\mathrm{OH},-\mathrm{CH},>\mathrm{CH}_{2},>\mathrm{C}=\mathrm{O},>\mathrm{NH},>\mathrm{C}=\mathrm{C}<$, and $\mathrm{C}=\mathrm{N}$. The absorption peaks of these groups in each band can be attributed to the absorption peak of the protein group [67].

The optical constants (the complex refractive index, CRI) of bioaerosols in the range of $0.24-14 \mu \mathrm{m}$ were discussed and analyzed, and the differences between the 


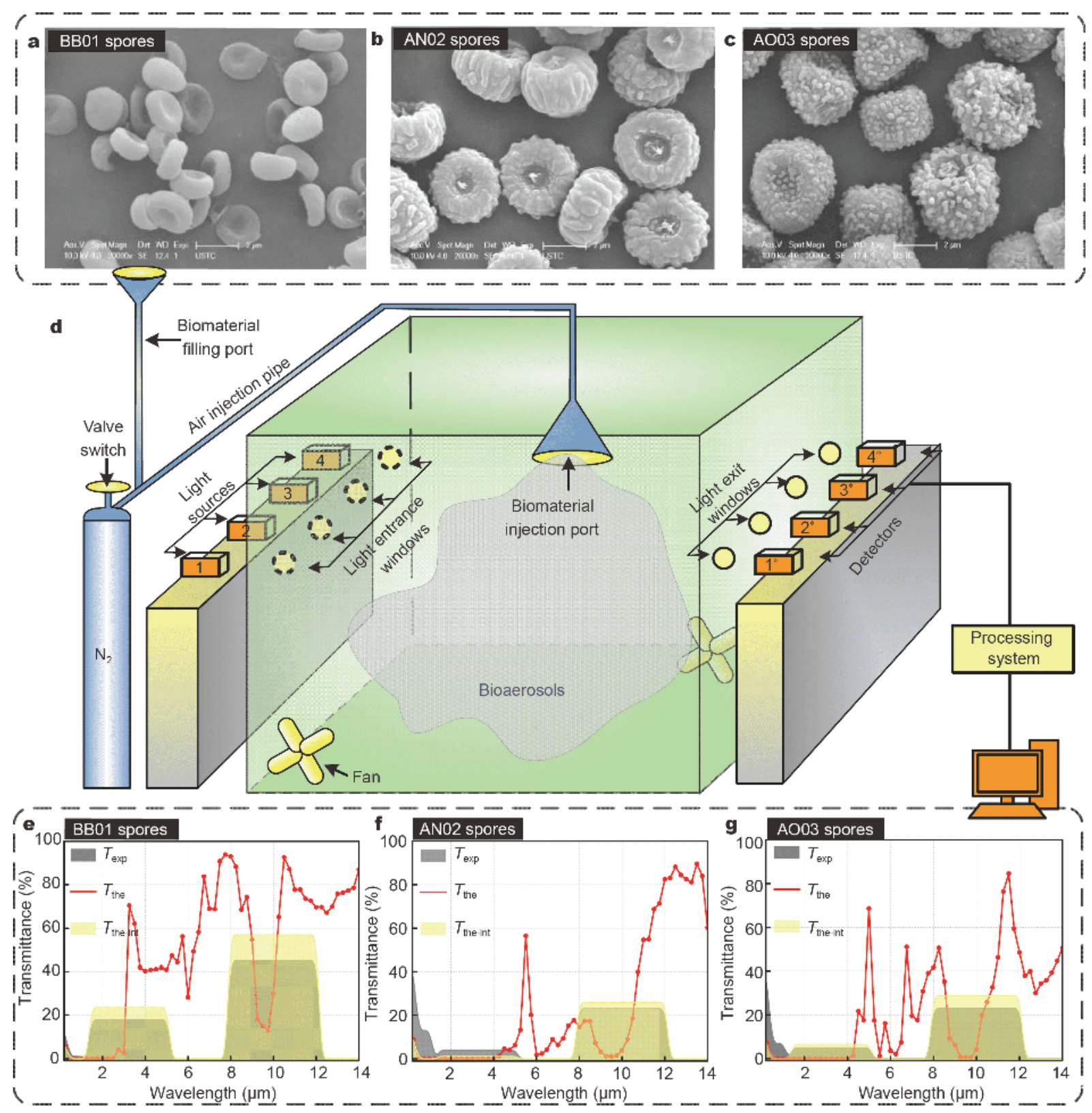

Figure 4 Transmittance experiment of bioaerosols in the smoke box. (a-c) Electron micrographs (20,000× magnification) of BB01 spores, AN02 spores and AO03 spores. (d) Smoke box schematic. The left part in the diagram is the bioaerosol injection system and the light sources; the right part is the detectors and signal processing system. Biomaterials in $(\mathrm{a}-\mathrm{c})$ are filled at the material filling port. The optical signal received by the detector is converted into the average transmittance of each test band after processing. (e-g) Grey shades show the actual measured average transmittance of each test band for three bioaerosols, marked as $T_{\text {exp }}$. Red spots represent theoretical transmittance values at each wavelength, marked as $T_{\text {the. }}$ Connecting the numerical values of theoretically calculated transmittance spots with curves is helpful for observing the trend and average values of the theoretical calculation transmittance in a certain band. Yellow-shaded parts represent the integral average value of theoretically calculated transmittance in the response detection bands, marked as $T_{\text {the-int }}$.

values in this paper and some literatures were compared. There are various methods for calculating the optical constants of materials, and reflection and transmission methods are common methods [33,68-70]. The transmission method is often used for the dilute solution, the thin film layer or the $\mathrm{KBr}$ tablet of the material to be tested. The condition for measuring the optical constant using the transmission method is that the light can penetrate the medium to be tested and the attenuation law in the medium conforms to Lambert Beer's law, and the 
material to be tested is a weakly absorbing substance. The reflection method is commonly used to measure the spectral reflectance of a bulk material or a material tablet that is directly pressed from a powdered material. In this case, the light cannot directly penetrate the object to be tested [71] or cannot penetrate the reference substrate under the object to be tested, and the material to be tested is a strong absorption material. It has been clearly pointed out in the literature $[70,72]$ that the optical constants of the film state and the bulk state of the same substance are completely different, and sometimes the difference even reaches several orders of magnitude [73]. In this paper, bioaerosols were sprayed in a smoke chamber to form a high-concentration aerosol smoke screen to study the wide-band shadowing effect of bioaerosol on electromagnetic waves. It can be seen from Fig. $2 b-d$ that the bioaerosol particles floating in the real state are very dense and non-spherical, and the attenuation of light in bioaerosols is caused by multiple scattering and absorption, which does not conform to Lambert Beer's law. On the other hand, in the literature [25], it is clearly pointed out that the attenuation of incident infrared light in the aerosol formed by Bacillus subtilis mainly depends on its absorption, and thus it can be seen that the strong absorption of bioaerosols cannot be ignored. In summary, in the context of the application of this paper, in order to match the experimental data of the smoke box as much as possible and reduce the error between theory and experiment, the spectral constant method should be used to calculate the optical constant of the bioaerosol. Therefore, the optical constants calculated in this paper are different from those of the same biological materials published in [20,26,74-81], which were obtained by the spectral transmission method. There is one more point to be noticed, even with the same research method, different material batches and experimental conditions will make the calculated optical constants different. This situation is evident in the series $[26,80,81]$. The measurement and calculation of the optical constants of materials have always been a heated discussion, and the methods are constantly improving. Fortunately, this paper focuses on the trend of the optical constants of bioaerosols in the $0.24-14 \mu \mathrm{m}$ band to demonstrate the broad-band extinction properties of bioaerosols.

The transmittance in Fig. $3 \mathrm{c}$ was simulated by using the Monte Carlo method and can be used to directly compare with the actual measured transmittance values obtained experimentally. Fig. 3d shows the extinction proportion of incident light by AN02 spores in the $240 \mathrm{~nm}$ to $14 \mu \mathrm{m}$ band. As shown by the data in Fig. 3d, when the aerosol density is $200 \mathrm{~cm}^{-3}$, the incident light in the $1-3 \mu \mathrm{m}$ band is essentially completely absorbed or scattered by the bioaerosol, and the transmitted light is relatively weak. This phenomenon is particularly noticeable when the density reaches $1,000 \mathrm{~cm}^{-3}$. The extinction ability of AN02 spores is relatively strong at 6.1 and $9.5 \mu \mathrm{m}$ and is weaker at 5.5, 8 and $12-14 \mu \mathrm{m}$, which is consistent with the changing nodes of the imaginary part of the complex refractive indexes in Fig. 3b. The extinction ability of AN02 spores in $240 \mathrm{~nm}$ to $14 \mu \mathrm{m}$ can be divided into absorption and scattering attenuation. Their corresponding proportions of the total extinction are shown in Fig. $3 e$ and $\mathrm{f}$. Bioaerosol particle density has very little effect on the change of the absorption and scattering proportions; thus, the black and red lines in Fig. 3e and $\mathrm{f}$ essentially coincide. In the $240 \mathrm{~nm}$ to $2.5 \mu \mathrm{m}$ band, scattering is relatively strong, which is related to the particle size of the primary bioaerosol particles. However, the extinction ability of AN02 spores is determined mainly by absorption. As shown in Fig. 3f, the absorption accounts for more than $90 \%$ (this data is obtained by averaging the absorption proportion of the entire band) of the total extinction. In some bands, such as $2.75-5 \mu \mathrm{m}$ and $6-10.5 \mu \mathrm{m}$ bands, the extinction effect is almost entirely due to the absorption. Such extinction ability dominated by absorption is not unique to AN02 spores. In fact, as shown in Figs S9 and S10, the calculation results for BB01 spores and AO03 spores also show the same characteristics. This research result has brought us new insight, consisting with the statement in reference [25]. Although there are many types of bioaerosols, and their extinction abilities vary in a wide range of wavelengths, absorption is the main reason for their extinction. Moreover, different bioaerosols exhibit similar strong extinction abilities at 6.1, 9.5 and 1-3 $\mu \mathrm{m}$.

As shown in Fig. 4e-g, the integral average of theoretically calculated transmittance (yellow shades) is smaller than the actual measured average transmittance (grey shades, see Fig. S11) for three bioaerosols of the ultraviolet and visible bands. This is due to the error caused by the large value of $|m| k d$ in the DDA calculation. According to the literature [82], when the value of $|m| k d$ is greater than 1 (see Fig. S12), the calculated value of the absorbing portion in the extinction action is overestimated, resulting in a theoretical calculation value smaller than the actual value. In Fig. 4e-g, compared with that of BB01 spores, the theoretical calculation data of AN02 spores and AO03 spores are more consistent with the actual measurements. In the calculation model of the extinction characteristics of the bioaerosol aggregated 
particle swarm, the original bioaerosol particles are assumed to be spherical. If the original particle is not spherical, it is necessary to convert the original particle into an equal volume sphere for calculation, giving rise to a certain error. Among the three materials used in the test, the original particles of $\mathrm{BB} 01$ spores are the least spherical, causing a larger error. The extinction performance of the three bioaerosols in the visible to NIR ranges is relatively strong. Both theoretical calculations and experimental measurements show this characteristic.

In conclusion, the presented results help us better understand the broadband extinction abilities of bioaerosols. When the content of bioaerosols in a specific area increases or decreases, the optical radiation characteristics of this area change accordingly, especially in the strong absorption band of bioaerosols, which provides new directions for the development of broadband light attenuation materials in UV to IR bands. Traditional aerosol extinction materials, such as copper powder, aluminium powder, graphite and red phosphorus, mostly have the shortcomings of single light shielding band, high cost, difficult degradation, chemical pollution, short circuit and so on. However, bioaerosols have not only a wide light shielding band, but also abundant sources, lower costs, easier degradation and less pollution. Compared with traditional aerosol extinction materials, biological aerosols have more application value in the field of broadband extinction materials. The findings in this study provide a platform for further exploitation of the advantages of bioaerosols while avoiding their disadvantages. The theoretical analysis can provide support for rapid detection and remote sensing detection of bioaerosols.

Received 27 November 2018; accepted 15 February 2019; published online 12 March 2019

1 Griffin DW. Atmospheric movement of microorganisms in clouds of desert dust and implications for human health. Clinical MicroBiol Rev, 2007, 20: 459-477

2 Gilbert Y, Duchaine C. Bioaerosols in industrial environments: a review. Can J Civ Eng, 2009, 36: 1873-1886

3 Liu W, Zhu X, Lei M, et al. A detailed procedure for CRISPR/Cas9mediated gene editing in Arabidopsis thaliana. Sci Bull, 2015, 60: 1332-1347

4 Wei K, Zheng Y, Li J, et al. Microbial aerosol characteristics in highly polluted and near-pristine environments featuring different climatic conditions. Sci Bull, 2015, 60: 1439-1447

5 Castillo JA, Staton SJR, Taylor TJ, et al. Exploring the feasibility of bioaerosol analysis as a novel fingerprinting technique. Anal Bioanal Chem, 2012, 403: 15-26

6 Fröhlich-Nowoisky J, Kampf CJ, Weber B, et al. Bioaerosols in the Earth system: Climate, health, and ecosystem interactions. Atmos
Res, 2016, 182: 346-376

7 Van Leuken JPG, Swart AN, Havelaar AH, et al. Atmospheric dispersion modelling of bioaerosols that are pathogenic to humans and livestock-A review to inform risk assessment studies. Microbial Risk Anal, 2016, 1: 19-39

8 Kenny CM, Jennings SG. Background bioaerosol measurements at mace head. J Aerosol Sci, 1998, 29: S779-S780

9 Reid JP, Bertram AK, Topping DO, et al. The viscosity of atmospherically relevant organic particles. Nat Commun, 2018, 9: 956

10 Després VR, Huffman JA, Burrows SM, et al. Primary biological aerosol particles in the atmosphere: a review. Tellus B-Chem Phys Meteor, 2012, 64: 15598

11 Matthias-Maser S, Obolkin V, Khodzer T, et al. Seasonal variation of primary biological aerosol particles in the remote continental region of Lake Baikal/Siberia. Atmos Environ, 2000, 34: 3805-3811

12 Jaenicke R. Abundance of cellular material and proteins in the atmosphere. Science, 2005, 308: 73

13 Huffman JA, Sinha B, Garland RM, et al. Size distributions and temporal variations of biological aerosol particles in the Amazon rainforest characterized by microscopy and real-time UV-APS fluorescence techniques during AMAZE-08. Atmos Chem Phys, 2012, 12: 11997-12019

14 Crutzen PJ. Geology of mankind. Nature, 2002, 415: 23

15 Adams KF, Hyde HA, Williams DA. Woodlands as a source of allergens. Allergy, 1968, 23: 265-281

16 Spänkuch D, Döhler W, Güldner J. Effect of coarse biogenic aerosol on downwelling infrared flux at the surface. J Geophys Res, 2000, 105: 17341-17350

17 Guyon P, Graham B, Roberts GC, et al. Sources of optically active aerosol particles over the Amazon forest. Atmos Environ, 2004, 38: 1039-1051

18 Makogon MM. Comparative analysis of spectroscopic methods for remote diagnostics of bioaerosols. Atmos Ocean Opt, 2011, 24: 123-132

19 Christesen SD, Merrow CN, Desha MS, et al. Ultraviolet fluorescence lidar detection of bioaerosols. SPIE Proc, 1994, 2222: 228237

20 Yabushita S, Wada K, Takai T, et al. A spectroscopic study of the microorganism model of interstellar grains. Astrophys Space Sci, 1986, 124: 377-388

21 Wickramasinghe NC, Wallis MK, Al-Mufti S, et al. The organic nature of cometary grains. Earth Moon Planet, 1988, 40: 101-108

22 Hoyle F, Wickramasinghe NC, Al-Mufti S. The ultraviolet absorbance of presumably interstellar bacteria and related matters. Astrophys Space Sci, 1985, 111: 65-78

23 Ligon DA, Wetmore AE, Gillespie PS. Simulation of the passive infrared spectral signatures of bioaerosol and natural fog clouds immersed in the background atmosphere. Opt Express, 2002, 10: 909

24 Gittins CM, Piper LG, Rawlins WT, et al. Passive and active standoff infrared detection of bio-aerosols. Field Analyt Chem Technol, 1999, 3: 274-282

25 Gurton KP, Ligon D, Kvavilashvili R. Measured infrared spectral extinction for aerosolized Bacillus subtilis var niger endospores from 3 to $13 \mu \mathrm{m}$. Appl Opt, 2001, 40: 4443-4448

26 Yabushita S, Wada K. The infrared and ultraviolet absorptions of micro-organisms and their relation to the Hoyle-Wickramashinghe hypothesis. Astrophys Space Sci, 1985, 110: 405-411

27 Wang $\mathrm{P}$, Liu $\mathrm{H}$, Zhao $\mathrm{Y}$, et al. Electromagnetic attenuation characteristics of microbial materials in the infrared band. Appl 
Spectrosc, 2016, 70: 1456-1463

28 Liu H, Wang $\mathrm{P}, \mathrm{Hu} \mathrm{Y}$, et al. Optimised fermentation conditions and improved collection efficiency using dual cyclone equipment to enhance fungal conidia production. Biocontrol Sci Tech, 2015, 25: $1011-1023$

29 Liu H, Zhao X, Guo M, et al. Growth and metabolism of Beauveria bassiana spores and mycelia. BMC Microbiol, 2015, 15: 267-279

30 Segal-Rosenheimer M, Linker R. Impact of the non-measured infrared spectral range of the imaginary refractive index on the derivation of the real refractive index using the Kramers-Kronig transform. J Quantitative Spectr Radiative Transfer, 2009, 110: 1147-1161

31 Booij HC, Thoone GPJM. Generalization of Kramers-Kronig transforms and some approximations of relations between viscoelastic quantities. Rheol Acta, 1982, 21: 15-24

32 Grosse P, Offermann V. Analysis of reflectance data using the Kramers-Kronig relations. Appl Phys A, 1991, 52: 138-144

33 Poelman D, Frederic Smet P. Methods for the determination of the optical constants of thin films from single transmission measurements: a critical review. J Phys D-Appl Phys, 2003, 36: 1850-1857

34 Zhao X, Hu Y, Gu Y. The infrared spectral transmittance of Aspergillus Niger spore aggregated particle swarm. SPIE Proc, 2015, 9678: 1717

35 Sun D, Hu Y, Gu Y, et al. Determination and model construction of microbes' complex refractive index in far infrared band. Acta Phys Sin, 2013, 62: 268-276

36 Li L, Hu Y, Chen W, et al. Measurement and analysis on complex refraction indices of pear pollen in infrared band. Spectrosc Spectr Anal, 2015, 35: 89-92

37 Sun D, Hu Y, Wang Y, et al. Sub-microstructures' influences on cell's scattering prosperities. Acta Phot Sin, 2013, 42: 710-714

38 Sun $\mathrm{D}, \mathrm{Hu} \mathrm{Y,} \mathrm{Gu} \mathrm{Y,} \mathrm{et} \mathrm{al.} \mathrm{Preparation} \mathrm{and} \mathrm{performance} \mathrm{testing} \mathrm{of}$ metallic biologic particles. Acta Phot Sin, 2013, 42: 555-558

39 Sun D, Hu Y, Li L. Test and analysis of infrared and microwave characteristics of metallic farinas. Infrared Laser Eng, 2013, 42: 2531-2535

40 Gu Y, Wang C, Yang L, et al. Infrared extinction before and after Aspergillus niger spores inactivation. Infrared Laser Eng, 2015, 44: 36-41

$41 \mathrm{Li} \mathrm{L}, \mathrm{Hu} \mathrm{Y}, \mathrm{Gu} \mathrm{Y}$, et al. Infrared extinction performance of Aspergillus niger spores. Infrared Laser Eng, 2014, 43: 2176-2180

42 Li L, Hu Y, Gu Y, et al. Infrared extinction performance of randomly oriented microbial-clustered agglomerate materials. Appl Spectrosc, 2017, 71: 2555-2562

$43 \mathrm{Gu}$ Y, Hu Y, Zhao X, et al. Discrimination of viable and dead microbial materials with Fourier transform infrared spectroscopy in 3-5 micrometers. Opt Express, 2018, 26: 15842

44 Zhao X, Hu Y, Gu Y. Transmittance of laser in the microorganism aggregated particle Swarm. Acta Optica Sin, 2015, 35: 222-228

45 Yurkin MA, Hoekstra AG. The discrete dipole approximation: An overview and recent developments. J Quantitat Spectr Rad Transfer, 2007, 106: 558-589

46 Li C, Xiong H. 3D simulation of the Cluster-Cluster aggregation model. Comput Phys Commun, 2014, 185: 3424-3429

47 Draine BT, Flatau PJ. Discrete-dipole approximation for scattering calculations. J Opt Soc Am A, 1994, 11: 1491-1499

48 Flatau PJ, Draine BT. Fast near field calculations in the discrete dipole approximation for regular rectilinear grids. Opt Express, 2012, 20: 1247-1252

49 Kinnunen M, Kauppila A, Karmenyan A, et al. Effect of the size and shape of a red blood cell on elastic light scattering properties at the single-cell level. Biomed Opt Express, 2011, 2: 1803

50 Dong J, Zhao JM, Liu LH. Effect of spine-like surface structures on the radiative properties of microorganism. J Quantit Spectr Radiat Transfer, 2016, 173: 49-64

51 Lee E, Heng RL, Pilon L. Spectral optical properties of selected photosynthetic microalgae producing biofuels. J Quantit Spectr Radiat Transfer, 2013, 114: 122-135

52 Lattuada M, Wu H, Morbidelli M. Radial density distribution of fractal clusters. Chem Eng Sci, 2004, 59: 4401-4413

53 Kozasa T, Blum J, Okamoto $\mathrm{H}$, et al. Optical properties of dust aggregates I. Wavelength dependence. Astron Astrophys, 1992, 263: 423-432

54 Kozasa T, Blum J, Okamoto $\mathrm{H}$, et al. Optical properties of dust aggregates II. Angular dependence of scattered light. Astron Astrophys, 1993, 276: 278-288

55 Min M, Dominik C, Hovenier JW, et al. The $10 \mathrm{~m}$ amorphous silicate feature of fractal aggregates and compact particles with complex shapes. Astron Astrophys, 2006, 445: 1005-1014

56 Huang C, Wu Z, Liu Y, et al. Effect of porosity on optical properties of aerosol aggregate particles. Acta Optica Sin, 2013, 33: 129001

57 Draine BT. The discrete-dipole approximation and its application to interstellar graphite grains. Astrophys J, 1988, 333: 848-872

58 Jacques SL. Modeling tissue optics-using Monte Carlo modeling a tutorial. SPIE Proc, 2008, 6854: 1-9

59 Wang L, Jacques SL, Zheng L. MCML-Monte Carlo modeling of light transport in multi-layered tissues. Comput Methods Programs Biomed, 1995, 47: 131-146

60 Zhao X, Hu Y, Gu Y, et al. Transmittance of laser in the microorganism aggregated particle swarm. Acta Phot Sin, 2015, 35: 0616001

61 Liu J, Zeng Y, Yang C. Light scattering study of biological cells with the discrete dipole approximation. Infrared Laser Eng, 2014, 43: 2204-2208

62 Jin S, Chen H. Near-infrared analysis of the chemical composition of rice straw. Industrial Crops Products, 2007, 26: 207-211

63 Cao S, Zhao Y. Application of molecular absorption spectrophotometric method to the determination of biologic macromolecular structures. Spectrosc Spect Anal, 2004, 24: 1197-1201

64 Lin X, Pan Y, Guo Y, et al. The study of cervical cancer cells model based on UV absorption spectrum. Spectrosc Spect Anal, 2009, 29: 2547-2550

65 Susi H, Byler DM. Fourier transform infrared study of proteins with parallel $\beta$-chains. Archives Biochem Biophys, 1987, 258: 465469

66 Li D, Yan C, Hu F, et al. Studied on simulation spectra of protein secondary structures by two-dimensional infrared correlation spectroscopy. J Light Scatt, 2013, 25: 417-422

67 Saito I, Sugiyama H, Matsuura T. Photochemical reactions of nucleic acids and their constituents of photobiological relevance. Photochem Photobiol, 1983, 38: 735-743

68 del Pozo JM, Díaz L. A comparison of methods for the determination of optical constants of thin films. Thin Solid Films, 1992, 209: 137-144

69 Li J, An W, Zhu T. The development of measurement and calculation model of the medium complex-refractive index. Energy Conserv Technol, 2017, 35: 214-219

70 Zhong D, Wang L, Yu Y. Optical constants measurement of thin film by spectrophotometry. J Liaoning Univ Natl Sci Ed, 1996, 23: 
$1-13$

71 Pekker D. A method for determining thickness and optical constants of absorbing thin films. Thin Solid Films, 2003, 425: 203-209

$72 \mathrm{Wu}$ Q. Ellipsometry of measuring for optical constant of an absorbing film. J Zhejiang Univ, 1982, 16: 1-7

73 Li C. Absorption and scattering of optical films. J Applied Optics, 1982, 3: 3-13

74 Bailey GF, Karp S, Sacks LE. Ultraviolet-absorption spectra of dry bacterial spores. J Bacteriol, 1965, 99: 984-987

75 Inagaki T. Optical absorptions of aliphatic amino acids in the far ultraviolet. Biopolymers, 1973, 12: 1353-1362

76 Tuminello PS, Arakawa ET, Khare BN, et al. Optical properties of Bacillus subtilis spores from 0.2 to $25 \mu \mathrm{m}$. Appl Opt, 1997, 36: 2818-2824

77 Arakawa ET, Tuminello PS, Khare BN, et al. Optical properties of horseradish peroxidase from 0.13 to $2.5 \mu \mathrm{m}$. Biospectroscopy, 1997, 3: 73-80

78 Emerson LC, Williams MW, Tang I, et al. Optical properties of guanine from 2 to $82 \mathrm{eV}$. Radiat Res, 1975, 63: 235-244

79 Hill SC, Doughty DC, Pan YL, et al. Fluorescence of bioaerosols: mathematical model including primary fluorescing and absorbing molecules in bacteria: errata. Opt Express, 2014, 22: 22817

80 Yabushita S, Wada K, Inagaki $\mathrm{T}$, et al. Photometric and photo accoustic measurement of the absorbance of micro-organisms and its relation to the micro-organism-grain hypothesis. Astrophys Space Sci, 1985, 117: 401-406

81 Hoyle F, Wickramasinghe NC, Al-Mufti S. The measurement of the absorption properties of dry micro-organisms and its relationship to astronomy. Astrophys Space Sci, 1985, 113: 413-416 82 Draine BT, Flatau PJ. User Guide for the Discrete Dipole Approximation Code. DDSCAT 7.3, 2013

Acknowledgements We thank Professor B. T. Draine of Princeton University for providing the main program of DDA. This work was supported by the National Natural Science Foundation of China (61271353 and 60908033), and the Natural Science Foundation of Anhui Province (1408085MKL47).

Author contributions $\mathrm{Hu} \mathrm{Y}$ and Zhao X conceived and designed the experiments. Zhao X, Wang $\mathrm{X}$ and Chen $\mathrm{X}$ performed the experiments. Zhao X analyzed the data. Gu Y, Wang P, Zheng Z and Dong X contributed reagents/materials/analysis tools. $\mathrm{Hu} \mathrm{Y}$ and Zhao $\mathrm{X}$ wrote the paper.

Conflict of interest The authors declare no competing interests.

Supplementary information Supporting material is available in the online version of the paper.

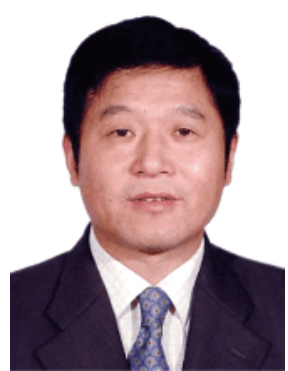

Yihua Hu received his BSc degree in radar engineering from the Electronic Engineering Institute of PLA (1983), MSc degree in circuits and systems from Xi'an University of Electronic Science and Technology (1988), and PhD degree in optics from Anhui Institute of Optics and Mechanics, Chinese Academy of Sciences (1997). He is now a professor of optical engineering at the National University of Defense Technology. His research interests include spatial information acquisition and processing technology, laser detection and imaging technology and photoelectric information and image processing.

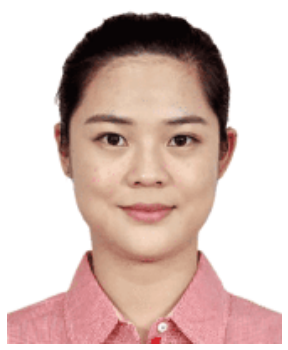

Xinying Zhao received her BSc degrees in information engineering and MSc in optical engineering from the National University of Defense Technology. She is now a PhD candidate at the National University of Defense Technology. Her research interests include the interaction of light and matter, particle scattering and functional materials.

\section{生物气溶胶具有显著的宽带消光能力}

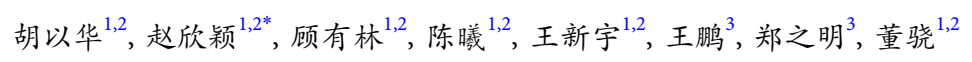

摘要 生物气溶胶是大气的重要组成部分, 因其吸收和散射效应, 可直接影响光辐射特性. 当前对于生物气溶胶是否具有宽波段消光特性 的研究还不够充分. 本文中, 我们测量了 12 种常见生物材料在 $240 \mathrm{~nm}-14 \mu \mathrm{m}$ 波段内的反射光谱, 并结合 $\mathrm{K}-\mathrm{K}$ 算法计算了不同生物气溶胶材 料的复折射率. 我们发现, 不同种质生物气溶胶的吸收峰具有共性, 位于约 $0.7,2.7,6.1$ 和 $9.5 \mu \mathrm{m}$ 处. 基于烟幕箱中生物气溶胶漂浮状态实际 结构的照片, 我们构建了模型计算 $240 \mathrm{~nm}-14 \mu \mathrm{m}$ 波长范围内生物气溶胶的消光能力. 以AN02孢子为例, 我们发现吸收作用占AN02孢子群 消光总量的 $90 \%$ 以. 此外, 我们对比了生物气溶胶理论计算透过率与大型烟幕箱实测透射率数据, 理论计算和实验验证都显示生物气溶 胶在紫外到红外波段具有显著的宽波段消光能力. 这一发现为宽波段消光材料的发展提供了新的研究方向. 